\title{
Perceived social support as a moderator between negative life events and depression in adolescence: implications for prediction and targeted prevention
}

\author{
Lence Miloseva $^{1} \cdot$ Tatjana Vukosavljevic-Gvozden $^{2} \cdot$ Kneginja Richter $^{1,3,4}$. \\ Vladimir Milosev ${ }^{1,5}$. Günter Niklewski ${ }^{1,3}$
}

Received: 27 March 2017 / Accepted: 12 May 2017 /Published online: 23 June 2017

(C) European Association for Predictive, Preventive and Personalised Medicine (EPMA) 2017

\begin{abstract}
Aim and background The role of the perceived social support in prevention of depression in adolescence still remains an insufficiently explored problem. By integrating the results of the previous studies of moderator role of perceived social support between negative life events and depression in adolescence we set up two goals. One is to determine whether perceived social support has moderator role in the sample consisted of clinical, subclinical, and control respondents. Another goal is to identify in which group the interaction effect is significant, i.e. the perceived social support acts as moderator.

Methods The sample consisted of 412 adolescents $(61.7 \%$ female and $38.3 \%$ male) aged $13-17$ years $($ mean $=15.70$, $\mathrm{SD}=1.22$ ). We applied: Data sheet for all respondents; Mini International Neuropsychiatric Interview; Multidimensional Scale of Perceived Social Support; Adolescent Life Events Questionnaire; Centre for Epidemiological Depression Scale. Results We have shown that the association between levels of depressive symptoms and negative life events changes as the value of the moderator variable perceived social support
\end{abstract}

Lence Miloseva

lence.miloseva@ugd.edu.mk

1 Faculty of Medical Science, Goce Delcev University, Ul. Krste Misirkov, bb, 2000 Stip, Republic of Macedonia

2 Faculty of Philosophy, Department of Psychology, University of Belgrade, Belgrade, Serbia

3 University Clinic for Psychiatry and Psychotherapy, Paracelsus Medical University, Nuremberg, Germany

4 Georg Simon University of Applied Science, Nuremberg, Germany

5 Clinical Hospital Centre, Stip, Macedonia changes. The finding that the moderating interaction effect was significant only in the subclinical group is particularly interesting.

Conclusions Taking into account that perceived social support moderates the association between negative stress events and levels of depression, we can propose a model for the prevention of depression, which will include perceived social support. However, future research with longitudinal design is required to verify the results.

Keywords Perceived social support · Moderator role . Negative life events · Depression · Adolescence · Prediction · Prevention

\section{Background}

\section{Psychosocial risk factors for depression}

Social support and negative life events (stressors)

Social support is an important variable that affects depressive symptomatology. A large number of studies show that depressed people have significant pervasive psychosocial difficulties. Specifically, higher levels of depressive symptoms are associated with lower levels of social support [1]. Perceived social support refers to the quality of emotional support provided by others. Research also suggests that rates of perceived social support are significantly associated with measures of reduced stress and psychological distress, as well as with improved measures of well-being [2, 3]. Yet, studies of social support in adolescents are mainly focused on the support of their families, and only a small number of studies examine the social support of peers [4]. 
Therefore, our study includes perceived social support from the family, friends, and significant others.

The review of literature and research has shown that social support (especially as perceived by adolescents) has been studied not only as a separate psychosocial risk factor for depression, but also as a factor that moderates the association between life events and depression, i.e. as a moderator variable, as it is treated in this study. One of them is the buffering model because it posits that support "buffers" (protects) persons from the potentially pathogenic influence of stressful events [5]. The idea that perceived social support protects against negative effects of stress and promotes psychological well-being has been supported by many recent studies [6-8]. Studies have confirmed the role of social support as a "buffer" between the negative stressful life events and depression, or that it is the "buffer" of undesirable effects of stress [9-12]. This is the reason why we are proposing to examine the justification for extending the model of cognitive vulnerability by introducing perceived social support, thus opening new questions about possibilities and needs for shifting towards an integrative approach to etiological models and new possibilities in research and applications in practice.

The conceptualization that stressful negative events have a significant impact on the development of symptoms of depression is common for all theories of cognitive vulnerability [13]. It has been found that the presence of negative life events is a reliable risk factor for the development of depressive symptoms in both sexes equally [14]. In adolescence, girls are exposed to a higher risk of developing symptoms of depression because it is assumed that they experience more negative life events (especially in interpersonal domains) and are more sensitive to stressful events, such as, for example, conflicts with peers $[15,16]$.

According to Calloway [13], cognitive vulnerability factors interact with negative life events, as predictors of elevated depressive symptoms in children (age 6-9 years) and in early adolescence (age 10-14 years). According to Hankin [17], if cognitive vulnerability factors continue to function in the presence of negative life events during adolescence, they remain cognitive risk factors for the development of depressive episodes later in adulthood. It is important to note that stressors and negative life events themselves, without the presence of cognitive vulnerability, are not strong predictors for depression in adolescence. Taking into account the diversity of negative life events, this study includes negative life events from the four domains relevant to adolescents: a) family and parents; b) romantic relationships; c) school and classes; and d) friends and social activities.

\section{The perceived social support in models of cognitive vulnerability to depression}

Models of cognitive vulnerability to depression in adolescence have been at the front position in research activities in the world. They define vulnerability as an internal and stable characteristic of an individual that predisposes a person to develop depression as a reaction to negative life events. It is important to emphasize that cognitive models are basically diathesis-stress models. The basic thesis is that depression stems from the interaction between cognitive vulnerability of a person (diathesis) and certain environmental conditions (stress), which serve as a trigger to activate this diathesis [18-20].

Whether subclinical depression is a clinically relevant condition and subclinically depressive adolescents are a risky and vulnerable group is still an insufficiently explored subject that arouses great interest. Subclinical depression is a condition in which a person has depressive symptoms, but does not meet the criteria for a depressive disorder [21]. Avenoli and associates [22] cite research and make comparisons between children and adolescents with subclinical and clinical major depression, indicating that a large percentage of children and adolescents with subclinical depressive symptoms show almost as many or more symptoms of depression compared with those with major depression, as well as the need for assistance and treatment.

From clinical point of view, subclinical depression is important for two reasons. First, subclinical depression is often a non-validating condition with significant psychological difficulties and need for treatment. The aim of the treatment is to reduce depressive symptoms and improve the quality of life. The second reason why subclinical depression is important from clinical point of view is the increased risk of developing major depression. Because of the increased risk of developing major depression in individuals with subclinical depression, the goal of interventions in subclinical depression is prevention of the occurrence of major depression. Although psychological treatment is effective [21], prevention is even more preferable.

In our opinion, one of the limitations of the models of cognitive vulnerability to depression is neglecting the importance of psychosocial factors, especially the perceived social support among adolescents. There is referent research in the world that tried to accentuate the importance of social environment in adolescence and its connection with depression [see 8]. The question of the moderating role of perceived social support in models of cognitive vulnerability to depression remains open, as well as the question what its role in the clinical, subclinical and control group of adolescents is. Perceived social support should be a part of the clinical evaluation of adolescents suffering from subclinical and clinical depression and a part of planning preventive and intervention strategies.

What do we actually expect to achieve with the proposed model for prevention of depression? The main hypothesis is that if it is shown that perceived social support moderates the relationship between negative stress events and levels of depression, then we can propose a model for the prevention of depression, which will include perceived social support. 
To date, researchers who have studied the effects of preventive interventions on depression, during the period of adolescence, generally have based their prevention strategies on cognitive-behavioral and/or interpersonal approaches [23]. Based on these approaches, we are proposing the model which focuses on the improvement of the perceived social support of adolescents who are at high risk of developing symptoms of depression. This may be done by promoting possibilities for supportive relationships and enhancing one's capability to use one's relationships as sources of social support. It is also important to change unhelpful cognitions of depressed adolescents and their overgeneralizing, which leads to the conclusion that all social others would be unsupportive in their expectations of a more optimistic future. The crucial factor for alleviating depression in times of high stress is the experience that someone, at least the psychotherapist, counselor, or teacher, as a significant other, shows concern about them.

\section{Current study}

\section{Method and material}

Starting from the literature and research, we have proposed a perceived social support (MPSS) as a moderator variable for which we expect to moderate the correlation between negative life events (ALEQ) and the level of depressive symptoms (CES-D), i.e. as a variable that defines the conditions under which the predictor variable of negative life events (ALEQ) is associated with the outcomes of the criterion variable (level of depressive symptoms).

\section{Participants}

This study is a cross-sequential, correlational study. In a 3year period, from January 2013 to January 2016, the main research was conducted in psychiatric clinics and primary and secondary schools in three main towns from three sociodemograhic regions in Macedonia (Skopje, Stip, Bitola) in order to make the sample as representative as possible. Three groups were formed: a clinical group, a subclinical group and a control group. All respondents in the sample were adolescents between the ages of 13 to 17 years. We used matching as a statistical technique taking into account age, sex, and sociodemographic region.

In order to satisfy the prerequisites for the statistical analysis, the clinical sample was planned to consist of 150 adolescents, from psychiatric wards and centers for mental health for children and adolescents from three clinics in Macedonia, 50 in each of the following centers: Stip, Skopje, and Bitola. However, only the data for 139 adolescents were taken into account in the final clinical sample. The criterion for inclusion was the fulfillment of the diagnostic criteria of DSM-IV-TR/
DSM-V [24, 25] for unipolar depression without psychotic features (Major Depressive Disorder, MDD). The instruments were given at admission to hospital to avoid the moderation effect of pharmacotherapy on the results.

The subclinical sample consisted of 133 respondents. It was formed from the initial sample of 720 adolescents from primary and secondary schools from the three sociodemographic regions: Stip, Skopje, and Bitola. The respondents from subclinical sample had the cutoff score for subclinical depression on the Centre for Epidemiological Depression Scale [26] over 16 and did not fulfill the criteria of the Mini International Neuropsychiatric Interview-Kid (M.I.N.I. -Kid) interview for major depression [27].

The control sample consisted of 140 respondents. It was formed from the remaining adolescents in the initial sample of 720 adolescents - those who had low scores on the Centre for Epidemiological Depression Scale below the cut-off score for subclinical depression, and did not fulfill the criteria of the M.I.N.I. -Kid interview for major depression. The exclusion criteria were: the presence of organic and psychotic disorders; somatic diseases; and IQ below 75 .

The final sample consisted of: the clinical group 139 (33.7\%) respondents, the subclinical group, 133 (32.3\%) respondents, and $140(34.0 \%)$ respondents in the control group. The average age of the entire sample was mean $=15.70$, $\mathrm{SD}=1.22$. The representation of female adolescents in the total sample was slightly higher than that of male respondents (61.7\% vs. $38.3 \%)$.

\section{Instruments}

The instruments that we used were translated into Macedonian. During translation, proofreaders of English and Macedonian language were consulted, and the method of double translation (back-translation) was applied.

Perceived social support was operationalized through The Multidimensional Scale of Perceived Social Support, MPSS [28]. This instrument is a 12-item self-descriptive scale designed to measure perceived social support of adolescents in three domains: family, friends, and significant others. It has three subscales, each consisting of four items that measure the three previously mentioned types of social support. Respondents need to circle the number that corresponds to their perception of social support. Statements are assessed on a seven-point scale (from 1- I completely agree to 7- I strongly disagree). The total score is obtained by summing and dividing by 12 . The four items that make up each subscale are summed and divided into four. Thus, the total score and the subscale scores range from $1-7$. Higher scores signify a greater level of perceived social support. The overall score in our study is marked with MPSS-total score of perceived social support. This scale showed good internal consistency [29]. The Multidimensional Scale of Perceived Social Support 
showed good internal consistency, $\alpha=.90$, on the Macedonian sample, based on a conducted pilot survey.

Negative life events (stressors) are best conceptualized along a continuum with some negative events that are more important than others (for example, death of parents; divorce of parents; low school achievement). They are operationalized through the Adolescent Life Events Questionnaire, ALEQ [18]. This questionnaire is a self-descriptive measure, a checklist, which measures a broad repertoire of negative life events that typically occur among adolescents (average age of 13-18 years). Negative life events are classified into four domains relevant to adolescents: a) family and parents, b) romantic relationships, c) schools and classes, and d) friends and social activities. Respondents were asked to read each event and to mark "yes" next to the event if it really happened to the respondent in the last 3 months. Overall scores are calculated by summing the answer is "yes" in every domain. Overall score in our study is marked with ALEQ - overall score of negative life events (stressors). Internal consistency is $\alpha=.94$. Testretest reliability for 2 weeks is $r=.65$ [18]. According to the pilot study conducted on the Macedonian sample, good measures of internal consistency are confirmed $(\alpha=.89)$.

The presence and level of depressive symptoms are operationalized through Centre for Epidemiological Depression Scale, CES-D [26]. Total score of 16 or higher is considered depressed. The CES-D is a 20 -item instrument developed by NIMH, Radloff [26], to detect major or clinical depression in adolescents and adults in community samples. The CES-D includes 20 items comprising 6 scales reflecting major facets of depression: depressed mood, feelings of guilt and worthlessness, feelings of helplessness and hopelessness, psychomotor retardation, loss of appetite, and sleep disturbance. The questions are easy to answer and cover most of the areas included in the diagnostic criteria for depression. Scores range from 0 to 60 , with depressive symptomatology indicated at a cutoff of 16 or above, as we used in this research as well.

The CES-D has been shown to be a good reliable measure for assessing the number, types, and duration of depressive symptoms with high internal consistency [26]. According to the pilot study conducted on the Macedonian sample, good measures of internal consistency are confirmed $(\alpha=.85)$. We also applied: Data sheet for all respondents; Mini International Neuropsychiatric Interview, M.I.N.I-Kid screen interview/ DSM-IV-TR [27].

\section{The procedure of the research}

After receiving the approval of the institutional ethics committee, the research was conducted in accordance with the ethical procedures and codes of psychological research, in clinics and schools in the three main centers of sociodemographic regions in the Republic of Macedonia (Stip, Eastern region; Skopje, Central Region; Bitola,
Western Region). According to the ethical procedure, all participants agreed to be involved in the research with guaranteed anonymity and signed informed consents.

\section{Results}

\section{Analysis of the results of the whole sample}

Table 1 shows arithmetic means and standard score deviations of all variables used in the study, as well as the size of the whole sample.

By using the hierarchical multiple regression analysis, we tested two models: the possibility of predicting level of depressive symptoms (CES-D) through negative life events (ALEQ) and perceived social support (MPSS), which was hypothesized in the first model, and the possibility of predicting the level of depressive symptoms (CES-D) through interaction of negative life events and perceived social support, which was hypothesized in the second model. In this way information can be obtained on whether there is a moderation of some of the listed predictors on the other predictors' associations with level of depressive symptoms. Hierarchical multiple regression is used to measure the effects of the moderator variable. To test moderation, we had to look specifically for an interaction effect between negative life events and perceived social support and whether such effect is significant in predicting level of depressive symptoms.

From the Table 2 we can see that both effects in both models, $\mathrm{R}^{2}=.825$ and $\mathrm{R}^{2}=.924$, and the model as a whole are statistically significant $(p<.001)$, which is a very high effect. The analysis has shown that it is possible to significantly predict the score of CES-D with ALEQ and MPSS with $82.5 \%$ accuracy $\left(\mathrm{R}^{2}=.825, p<.001\right)$. Adding their interactions into the model increases overall prediction to $92.4 \% ; \mathrm{R}^{2}$ $=.924$ accuracy which is very high effect $(p<.001)$. Prediction increase is thus $10 \%\left(\mathrm{R}^{2}\right.$ change $\left.=.100 ; p<.001\right)$ and is statistically significant. So, with the inclusion of the interaction effect in the model and with checking whether such interaction is actually significant, the variance of the criteria of depression can be explained better than before.

The Table 3 gives us the details from which we can see that $\mathrm{F}=964.132$ for the first model and $\mathrm{F}=1676.916$ are statistically significant, $p<.001$. This confirms our expectations that

Table 1 Descriptive statistics

\begin{tabular}{lrrr}
\hline & M & \multicolumn{1}{l}{ SD } & N \\
\hline CES-D & 26.599 & 18.690 & 412 \\
ALEQ & 37.388 & 15.412 & 412 \\
MPSS & 3.628 & 1.842 & 412 \\
ALEQ_MPSS_interaction & 108.478 & 23.978 & 412 \\
\hline
\end{tabular}


Table 2 Significance of the change of prediction

\begin{tabular}{lllllllllll}
\hline Model & $\mathrm{R}$ & $\mathrm{R}^{2}$ & Adjusted $\mathrm{R}^{2}$ & \multirow{2}{*}{$\begin{array}{l}\text { Std. Error of } \\
\text { the estimate }\end{array}$} & & & & & & \multicolumn{2}{l}{ Change statistics } \\
\cline { 6 - 9 } & & & & & $\mathrm{R}^{2}$ Change & F Change & df1 & df2 & Sig. F Change \\
\hline 1 & $.908^{\mathrm{a}}$ & .825 & .824 & 7.837 & .825 & 964.132 & 2 & 409 & .000 \\
2 & $.962^{\mathrm{b}}$ & .925 & .924 & 5.137 & .100 & 543.732 & 1 & 408 & .000 \\
\hline
\end{tabular}

${ }^{\text {a }}$ Predictors: (Constant), MPSS, ALEQ

${ }^{\mathrm{b}}$ Predictors: (Constant), MPSS, ALEQ, ALEQ_MPSS_interaction the first and second model predict the scores on CES-D on a statistically significant level.

Based on Table 4, we can see that MPSS stands out as a significant predictor in the first model $(\beta=-.840, t=-11.377$, $p<.001$, high score on MPSS leads to a lower score on the CES-D), and in the second model ALEQ stands out $(\beta=.754$, $t=13.333, p<.001$, high score on ALEQ leads to a higher score on the CES-D) and the interaction ALEQ and MPSS ( $\beta=-.384, t=-23.318, p<.001$, high interaction of ALEQ and MPSS leads to a lower score on CES-D). The fact is that in the first model MPSS is significant and that in the second it is not, but the interaction of MPSS with ALEQ argues in favor of the statement that MPSS moderates the interaction of ALEQ with CES-D with level of depressive symptoms.

So, we can see that the correlation between level of depressive symptoms and negative life events changes as the value of the moderator variable perceived social support changes, and this confirms that perceived social support is a moderator variable and that it moderates the association between negative life events and level of depressive symptoms.

\section{Analysis of the results within each subgroup of the sample separately}

In order to answer the second research question, in which of the examined subgroups of the sample the perceived social support is a moderator variable and whether it moderates the association between negative life events and level of

Table 3 ANOVA - Significance of prediction

\begin{tabular}{|c|c|c|c|c|c|c|}
\hline \multicolumn{2}{|c|}{ Model } & \multirow{2}{*}{$\begin{array}{l}\text { Sum of Squares } \\
118445.659\end{array}$} & \multirow{2}{*}{$\frac{\mathrm{df}}{2}$} & \multirow{2}{*}{$\begin{array}{l}\text { Mean Square } \\
59222.830\end{array}$} & \multirow{2}{*}{$\begin{array}{l}\mathrm{F} \\
964.132\end{array}$} & \multirow{2}{*}{$\frac{p}{.000}$} \\
\hline 1 & Regression & & & & & \\
\hline & Residual & 25123.260 & 409 & 61.426 & & \\
\hline & Total & 143568.920 & 411 & & & \\
\hline \multirow[t]{3}{*}{2} & Regression & 132798.775 & 3 & 44266.258 & 1676.916 & .000 \\
\hline & Residual & 10770.145 & 408 & 26.397 & & \\
\hline & Total & 143568.920 & 411 & & & \\
\hline
\end{tabular}

Significant at the $p<.001$ level

a. Predictors: (Constant), MPSS, ALEQ

b. Predictors: (Constant), MPSS, ALEQ, ALEQ_MPSS_interaction

c. Dependent Variable: CES-D depressive symptoms, we conducted the same procedure of hierarchical multiple regression analysis for each subgroup separately.

Table 5 shows the significance of prediction coefficients of the level of depression in the two models, through negative life events, perceived social support, and their interactions. Here follows the analysis of results by groups.

Based on standardized regression coefficients $(\beta)$, the analysis in the clinical group has shown that significant predictors in the first model are: MPSS $(\beta=-.529, t=-8.123, p<.001$, a low score on MPSS leads to a higher score on CES-D) and ALEQ $(\beta=.378, t=5.803, p<.001$, a high score on ALEQ leads to a higher score on CES-D). Based on the regression coefficients $(\beta)$, we can conclude that there are no major differences and changes in MPSS in the second model ( $\beta=-.528, t=-8.096, p<.001$, a low score on MPSS leads to a higher score on CES-D) and ALEQ $(\beta=.368, t=5.529$, $p<.001$, a high score on ALEQ leads to a higher score on CES-D). An interesting fact is that the interaction between ALEQ and MPSS in the clinical group is not significant.

In the subclinical group, the significant predictors in the first model are: MPSS $(\beta=-.529, t=-8.341, p<.001$, a low score on the MPSS leads to a higher score on CES-D) and ALEQ ( $\beta=.444, t=6.999, p<.001$, a high score on ALEQ leads to a higher score on CES-D). In the second model, there is no great difference in the size of beta for both predictors MPSS ( $\beta=-.563, t=-8.755, p<.001$, a low score on the MPSS leads to a higher score on CES-D) and ALEQ ( $\beta=.424$, $t=6.723, p<.001$, a high score on ALEQ leads to a higher score on CES-D). An interesting finding is that only in the subclinical group the interaction between negative life events and perceived social support proved to be significant (ALEQ_MPSS_interaction, $\beta=-.145, t=-2.228, p<.05$ ). Furthermore, we notice that the beta for ALEQ in the subclinical group is the greatest compared with the clinical and control group in both models. The same applies to the size of the beta for MPSS for the subclinical group compared with the clinical and control group.

In the control group, the predictors ALEQ and MPSS are significant, but judging by the sizes of the beta, the sizes of beta are still the lowest compared with the clinical and subclinical group. Below is an overview of the sizes of the beta. In the first model, for the predictor MPSS, $\beta=-.441, t=-6.272$, 
Table 4 Significance of regression coefficients

\begin{tabular}{|c|c|c|c|c|c|c|c|c|c|}
\hline \multicolumn{2}{|c|}{ Model } & \multirow{3}{*}{$\frac{B}{54.276}$} & \multirow{3}{*}{$\frac{\text { Std. Error }}{6.015}$} & \multirow{3}{*}{ Beta } & \multirow{3}{*}{$\begin{array}{l}\mathrm{t} \\
9.024\end{array}$} & \multirow{3}{*}{$\begin{array}{l}\mathrm{p} \\
.000\end{array}$} & \multicolumn{3}{|l|}{ Correlations } \\
\hline & & & & & & & Zero-order & Partial & Part \\
\hline \multirow[t]{3}{*}{1} & (Constant) & & & & & & & & \\
\hline & ALEQ & .087 & .089 & .071 & .967 & .334 & .877 & .048 & .020 \\
\hline & MPSS & -8.520 & .749 & -.840 & $-\mathbf{1 1 . 3 7 7}$ & .000 & -.908 & -.490 & -.235 \\
\hline \multirow[t]{4}{*}{2} & (Constant) & 27.843 & 4.103 & & 6.786 & .000 & & & \\
\hline & ALEQ & .914 & .069 & .754 & 13.333 & .000 & .877 & .551 & .181 \\
\hline & MPSS & -.816 & .592 & -.080 & -1.380 & .168 & -.908 & -.068 & -.019 \\
\hline & ALEQ_MPSS_interaction & -.299 & .013 & -.384 & -23.318 & .000 & -.496 & -.756 & -.316 \\
\hline
\end{tabular}

a. Dependent Variable: CES-D

Significant predictors are marked in bold and shown in the regression coefficients table

Significant at the $p<.001$ level

$p<.001$ (a low score on MPSS leads to a higher score on CES-D) and for the ALEQ, $\beta=.358, t=5.086, p<.001$ (a high score on ALEQ leads to a higher score on CES-D). In the other model, for the predictor MPSS, $\beta=-.437, t=-6.189$, $p<.001$ (a low score on MPSS leads to a higher score on CES-D) and for the ALEQ, $\beta=.357, t=5.070, p<.001$ (a high score on ALEQ leads to a higher score on CES-D).

This finding confirms that MPSS is the moderator variable and that the perceived social support moderates the association between negative life events and level of depression symptoms. So, we can see that only in the subclinical group the association between negative life events, ALEQ, and levels of depression, CES-D changes with the changes of the values of the moderator variables of perceived social support, MPSS.

\section{Discussion}

The results have shown that the correlation between levels of depressive symptoms and negative life events changes as the value of the perceived social support changes. Therefore, we can conclude that the perceived social support is a significant predictor and moderator variable, which functions as a "stress buffer" variable between negative stressful life events and the level of depressive symptoms. A more thorough analysis revealed that the moderating interaction effect between negative life events and perceived social support was significant only in the subclinical group. This finding is particularly important because of its various implications for prediction and targeted prevention. In order to be precise, firstly we shall discuss the analysis of the results of the whole sample, and secondly the
Table 5 The significance of regression coefficients of prediction level of depressive symptoms (CES-D) in both models, through negative life events (ALEQ), perceived social support (MPSS) and their interactions

\begin{tabular}{|c|c|c|c|c|c|c|c|}
\hline \multirow{2}{*}{$\begin{array}{l}\text { Group } \\
\text { Clinical }\end{array}$} & \multicolumn{2}{|c|}{ Model } & \multirow{2}{*}{$\begin{array}{c}\text { Beta } \\
.378\end{array}$} & \multirow{2}{*}{$\frac{\mathrm{t}}{5.803}$} & \multirow{2}{*}{$\begin{array}{l}\mathrm{p} \\
.000\end{array}$} & \multirow{2}{*}{$\begin{array}{l}\mathrm{r} \\
. \mathbf{3 7 8}\end{array}$} & \multirow{2}{*}{$\begin{array}{l}\text { Pr } \\
.445\end{array}$} \\
\hline & 1 & ALEQ & & & & & \\
\hline & & MPSS & -.529 & -8.123 & .000 & -.529 & -.572 \\
\hline & 2 & ALEQ & .368 & 5.529 & .000 & .378 & .430 \\
\hline & & MPSS & -.528 & -8.096 & .000 & -.529 & -.572 \\
\hline & & ALEQ_MPSS_interaction & -.050 & -.758 & .450 & -.132 & -.065 \\
\hline \multirow[t]{5}{*}{ Subclinical } & 1 & ALEQ & .444 & 6.999 & .000 & .444 & .523 \\
\hline & & MPSS & -.529 & -8.341 & .000 & -.529 & -.590 \\
\hline & 2 & ALEQ & .424 & 6.723 & .000 & .444 & .509 \\
\hline & & MPSS & -.563 & -8.755 & .000 & -.529 & -.610 \\
\hline & & ALEQ_MPSS_interaction & -.145 & -2.228 & .028 & -.069 & -.193 \\
\hline \multirow[t]{5}{*}{ Control } & 1 & ALEQ & .358 & 5.086 & .000 & .358 & .399 \\
\hline & & MPSS & -.441 & -6.272 & .000 & -.441 & -.472 \\
\hline & 2 & ALEQ & .357 & 5.070 & .000 & .358 & .399 \\
\hline & & MPSS & -.437 & -6.189 & .000 & -.441 & -.469 \\
\hline & & ALEQ_MPSS_interaction & -.062 & -.879 & .381 & -.098 & -.075 \\
\hline
\end{tabular}

Significant predictors are marked in bold and significant at the $p<.001$ level. Only ALEQ_MPSS_interaction in subclinical group is significant at the level $p<.05$ 
analysis of the results within each subgroup of the sample separately.

Using hierarchical multiple regression analysis on the whole sample we tested two models: that it is possible to predict the level of depressive symptoms through negative life events and perceived social support, and that it is possible to predict the level of depressive symptoms through the interaction between negative life events and perceived social support.

The analysis showed that both effects in both of the mentioned models and the model as a whole are statistically significant and that it is possible to significantly predict the level of depressive symptoms by means of negative life events and perceived social support with $82.5 \%$. Adding their interactions into the model increases total prediction to $92.4 \%$ accuracy, which is a very high effect. The increase of prediction is therefore $10 \%$ and is statistically significant. So, with the inclusion of the interaction effects in the model, and checking whether such interaction is actually significant, the variance of depression criteria can be explained better than before.

Based on regression coefficients we have seen that in the first model the perceived social support stands out as a significant predictor, i.e. a highly perceived social support leads to a low level of depressive symptoms. In the second model, negative life events stand out, i.e. more negative life events lead to a higher level of depressive symptoms and higher interaction of negative life events and perceived social support leads to a low level of depressive symptoms. To our knowledge, there have been no other similar studies conducted so far, so these results are important and worth further exploring.

What do these results further mean? The fact that in the first model perceived social support is significant, but in the second model it is not, but the interaction of perceived social support with negative life events is significant, argues in favor of the fact that the perceived social support moderates the association of negative life events with the level of depressive symptoms. These findings are consistent with the determination of social support as a "stress buffer" variable. The determination of social support as a "stress buffer" suggests that social support provides an individual with protection against potential stress events. It helps an individual to reduce the amount of the experienced stress and to deal better with stress in life situations [8]. Long ago, Cohen and Willis [5] stressed that social support can play a role from two different aspects of the causal chain, linking stress with the effects of stress on an individual and at the same time suggesting that support can intervene between stressful events and stress reactions with preventing the estimate of stress reaction. In other words, it is possible that individuals do not experience the potential threat as stress, as a result of the belief that they have adequate resources by which they would be able to withdraw and protect themselves from the threat.

Furthermore, a more thorough analysis determined that only in the subclinical group the association between negative life events and level of depressive symptoms changes with the changes of the values of the perceived social support. This finding is particularly interesting and worth future studying because we have not met the research that explores this problem so far. It has many implications for the creation of specific prevention programs that would target members of a subgroup who are at higher risk for disorder.

\section{Conclusions}

It can be concluded that we have shown that the association between negative life events and the level of depressive symptoms changes with the change of perceived social support values. This confirms our expectations that perceived social support is a moderator variable and that it moderates the association between negative life events and the level of depressive symptoms. The findings are in line with the determination of social support as a "stress buffer" variable, which further suggests that perceived social support in adolescence provides protection against potential stressful events.

Furthermore, from a therapeutic point of view, our results suggest that the inclusion of perceived social support as a psycho-social variable in cognitive-behavioral etiological models of depression is justified. We find that the results relating to the subclinical group are of particular importance as they offer the first evidence of the moderating role of perceived social support between negative life events and levels of symptoms of depression. To our knowledge, no empirical research exists addressing question of implications of moderator role of perceived social support in group with subclinical depression. This makes our findings of great importance.

The results obtained will give a contribution to the overall scientific knowledge in the prediction and identification of high-risk, vulnerable subclinical group for depression, which will enable the creation of a specific model for early prediction, diagnosis and rational treatment, and thus a better longterm prognosis and improvement in the quality of adolescents' lives, which gives practical meaning to this paper.

The greatest value of the results is their implication for creating a model for the prevention of depression in adolescence. Support from psychotherapists or counselors is essential for the therapy to be successful, but involving support from family members, peers or significant other in the course of preventive interventions will further facilitate improvement. On the other hand, subjective appraisals of whether family, friends, or significant others will provide help are based largely on adolescents' assessments of the quality of their social relations. Therefore, prevention programs such as interpersonal communication and social skills training are as important as therapeutic treatments. The value of such prevention programs in enhancing the quality of social relations should not be ignored and underestimated. 
This study has some limitations. The study is a correlational, cross-sectional study. For introducing dynamism in the research itself, which is not enabled with this model of transactional design, we suggest inclusion of longitudinal dynamic dimensions as implications for further research that allow rigorous testing. In addition to the self-reporting measures of cognitive vulnerability and depression and quantitative data, we should add the interview and the reports from parents and teachers, as well as the qualitative data analysis.

\section{Expert recommendation}

It is evident that a paradigm shift is needed to move from "reactive" to "predictive, preventive, and personalized medicine" as a new philosophy, promoting an integrated approach combining advantages of individual bio/medical fields and consolidating a multi-professional collaboration [30].

In our opinion, future research should continue establishing an empirical basis for the prevention of depression, especially in subclinical adolescents who are at high risk of developing clinical features of depression. When creating a model of prevention, we find that it is of utmost importance to take into consideration the moderating power of perceived social support and resources that are available to clinicians, families, and schools. What will make this proposed model of prevention useful is: widespread use, the opportunity to be assessed and the capacity to deliver long term outcomes. We also need to take into account that the selected and indicated prevention approaches appear to be more effective than universal prevention approaches [23]. Selective prevention programs target members of a subgroup who are at a higher risk for disorder. Finally, indicated prevention programs target all people who display subclinical signs or symptoms of a given disorder. These approaches have been found to be helpful in the treatment of depression and recently have been evaluated to determine whether they may be useful in preventing depression in adolescence [see 31].

Based on contemporary research $[30,32]$ we believe that the new paradigm - predictive, preventive, and personalized medicine - offers new and greater possibilities for prediction and prevention of depression in adolescence.

\section{Abbreviations}

MPSS, perceived social support; ALEQ, negative life events; CES-D, level of depressive symptoms; MDD, Major Depressive Disorder; DSM-IV-TR/DSM-V, Diagnostic and Statistical Manual of Mental Disorders DSM-IV-TR (4th ed. Text Revision)/ Diagnostic and Statistical Manual of Mental Disorders, DSM-V (5th ed. Text Revision); NIMH, National Institute for Mental health; M.I.N.I.-Kid interview, Mini International Neuropsychiatric Interview; IQ, Intelligence quotient; ANOVA, analysis of variance.
Acknowledgements The study was partly supported by project No. 2502-261 Clinical and subclinical depression among adolescents, Goce Delcev University, Stip, Republic of Macedonia. This work would not have been possible without the professional support of all my colleagues from clinics and schools in Macedonia and Faculty of Medical Science, Goce Delcev University, Stip. We are thankful for that support.

Authors' contributions LM substantially contributed to the conception and design, acquisition of the data, and analysis and interpretation of the data; has been involved in drafting the manuscript or revising it critically; and agrees to be accountable for all aspects of this paper. TVG participated in the design of the study, contributed to the analysis and interpretation of the data; revising the manuscript critically. VM helped in the acquisition of the data, performed statistical analysis, and helped to draft the manuscript. KR and GN proof-read and make corrections to manuscript. All authors read and approved the final manuscript.

\section{Compliance with ethical standards}

Ethical approval Ethical approval was obtained from the Faculty of Medical Science, Goce Delcev University, Stip, R. Macedonia.

Competing interests The authors have declared that no competing interests exist.

Funding/financial support The authors have no funding to report.

Conflict of interest The authors declare that they have no conflict of interest.

\section{References}

1. Kaltiala-Heino R, Rimpelä M, Rantanen P, Laippala P. Adolescent depression: the role of discontinuities in life course and social support. J Affect Disord. 2001;64(2-3):155-66.

2. Yap MB, Devilly GJ. The role of perceived social support in crime victimization. Clin Psychol Rev. 2004;24(1):1-14.

3. Gjesfjeld CD, Greeno CG, Kim KH, Anderson CM. Economic stress, social support, and maternal depression: is social support deterioration occurring? Soc Work Res. 2010;34(3):135-43.

4. Colarossi LG, Eccles JS. A prospective study of adolescents' peer support: gender differences and the influence of parental relationships. J Youth Adolesc. 2000;29(6):661-78.

5. Cohen S, Wills TA. Stress, social support, and the buffering hypothesis. Psychol Bull. 1985;98(2):310-57.

6. Dahlem NW, Zimet GD, Walker RR. The multidimensional scale of perceived social support: a confirmation study. J Clin Psychol. 1991;47:756-61.

7. Brissette I, Scheier MF, Carver CS. The role of optimism in social network development, coping, and psychological adjustment during a life transition. J Pers Soc Psychol. 2002;82(1):102-11.

8. Monroe SM, Slavich GM, Georgiades K. The social environment and depression: the roles of life stress. In: Gotlib IH, Hammen CL, editors. Handbook of depression. New York: Guilford Press; 2014.

9. Bliese PD, Britt TW. Social support, group consensus, and stressorstrain relationships: social context matters. J Organ Behav. 2001;22: 425-326.

10. Cankaya B. Psychosocial factors, maladaptive cognitive schemas, and depression in young adults: an integration. [Electronic Thesis or Dissertation]. Blacksburg: Polytechnic Institute and State University; 2002. 
11. Clara IP, Cox BJ, Enns MW, Murray LT, Torgrude LJ. Confirmatory factor analysis of the multidimensional scale of perceived social support in clinically distressed and student samples. J Pers Assess. 2003;81(3):265-70.

12. Uchino BN. Understanding the links between social support and physical health: a lifespan perspective with emphasis on the separability of perceived and received support. Perspect Psychol Sci. 2009;4(3):236-55.

13. Calloway SC. Cognitive vulnerabilities, negative life events, and depressive symptoms in young adolescents. [Electronic Thesis or Dissertation]. Nashville: Graduate School of Vanderbilt University; 2010.

14. Ge X, Lorenz FO, Conger RD, Elder GH, Simons RL. Trajectories of stressful life events and depressive symptoms during adolescence. Dev Psychol. 1994;30(4):467-83.

15. Hankin BL, Abramson LY. Development of gender differences in depression: description and possible and explanations. Ann Med. 1999;31(6):372-9.

16. Shih JH, Eberhart NK, Hammen CL, Brennan PA. Differential exposure and reactivity to interpersonal stress predict sex differences in adolescent depression. J Clin Child Adolesc Psychol. 2006;35(1):103-15.

17. Hankin BL. Adolescent depression: Description, causes, and interventions. Epilepsy Behav. 2006;8:102-14.

18. Hankin BL, Abramson LY. Measuring cognitive vulnerability to depression in adolescence: reliability, validity, and gender differences. J Child Adolesc Clin Psychol. 2002;31(4):491-504.

19. Abela JRZ. The hopelessness theory of depression: a test of the diathesis-stress and causal mediation components in third and seventh grade children. J Abnorm Child Psychol. 2001;29(3):241-54.

20. Abela JRZ, Hankin BL, editors. Handbook of child and adolescent depression. New York: Guilford Press; 2008.

21. Cuijpers P, Smit F. Subklinische depressie: een klinisch relevante conditie? Tijdschr Psychiatr. 2008;50(8):519-28.
22. Avenevoli S, Knight E, Kessler RC, Merikangas KR. Epidemiology of depression in children and adolescents. In: Abela JRZ, Hankin BL, editors. Handbook of depression in children and adolescents. New York: Guilford Press; 2008.

23. Gillham JE, Shatte AJ, Freres DR. Preventing depression: a review of cognitive-behavioral and family interventions. Appl Prev Psychol. 2000;9:63-88.

24. American Psychiatric Association. Diagnostic and statistical manual of mental disorders, DSM-IV -TR (4th ed. Text Revision). Washington, DC: Author; 2000.

25. American Psychiatric Association. Diagnostic and statistical manual of mental disorders, DSM-V (5th ed. Text Revision). Washington, DC: Author; 2013.

26. Radloff LS. The CES-D scale: a self-report depression scale for research in the general population. Appl Psychol Meas. 1977;1: 385-401.

27. Sheehan D V, Lecrubier Y. M.I.N.I. SCREEN 5.0.0/English version/DSM-IV July/1/06. Florida: University of South FloridaTAMPA, USA; 2001/2006.

28. Zimet G, Dahlem N, Zimet S, Farley G. The multidimensional scale of perceived social support. J Pers Assess. 1988;52(1):30-41.

29. Canty-Mitchell J, Zimet GD. Psychometric properties of the multidimensional scale of perceived social support in urban adolescents. Am J Community Psychol. 2000;28:391-400.

30. Golubnitschaja O, Baban B, Boniolo G, Wang W, Bubnov R, Kapalla M, et al. Medicine in the early twenty-first century: paradigm and anticipation - EPMA position paper 2016. EPMA J. 2016;7:23.

31. Gladstone TRG, Beardslee WR, O'Connor EE. The prevention of adolescent depression. Psychiatr Clin N Am. 2011;34(1):35-52.

32. Veauthier C, Hasselmann H, Gold SM, Paul F. The berlin treatment algorithm: recommendations for tailored innovative therapeutic strategies for multiple sclerosis-related fatigue. EPMA J. 2016;7:25. 